\title{
Hadroproduction of a charged vector boson pair in association with a b-quark pair at NLO accuracy matched with parton shower
}

\section{Zoltán TRÓCSÁNYI*†}

Institute of Physics, University of Debrecen, H-4010 Debrecen P.O.Box 105, Hungary

E-mail: zoltan.trocsanyi@cern.ch

Maria Vittoria GARZELLI

II Institute for Theoretical Physics, University of Hamburg,, Hamburg, , Germany

E-mail: garzelli@to.infn.it

\section{Adam KARDOS}

MTA-DE Particle Physics Research Group, Debrecen, Hungary

E-mail: kardos.adamescience.unideb.hu

\begin{abstract}
We present simulated events for the process $p p \rightarrow W^{+} W^{-} \mathrm{b} \overline{\mathrm{b}} \rightarrow e^{+} v_{e} \mu^{-} \bar{v}_{\mu} \mathrm{b} \overline{\mathrm{b}}+X$ that give kinematic distributions at NLO QCD accuracy and can be further evolved by Shower Monte Carlo simulations. We include all resonant and non-resonant contributions. This is achieved by fully taking into account the effect of off-shell t-quarks and off-shell vector bosons in the complex mass scheme. We also present a program called Decayer that can be used to let the t-quarks present in the event files for $p p->\mathrm{t} \overline{\mathrm{t}}+X$ processes decay including both the finite width of the $\mathrm{t}$-quarks and spin correlations. This work is part of a bigger project in which we have provided similar events for the following final states: (i) $\mathrm{t}+\overline{\mathrm{t}}$, (ii) $\mathrm{t}+\overline{\mathrm{t}}+H / Z / W$, (iii) $\mathrm{t}+\overline{\mathrm{t}}+\mathrm{b}+\overline{\mathrm{b}}$, (iv) $\mathrm{t}+\overline{\mathrm{t}}+$ jet (v) $\mathrm{t}+\overline{\mathrm{t}}+\operatorname{photon}(\mathrm{s})$.
\end{abstract}

The European Physical Society Conference on High Energy Physics 22-29 July 2015

Vienna, Austria

\footnotetext{
${ }^{*}$ Speaker.

$\dagger$ This research was supported by the Swiss National Science Foundation (grant SCOPES IZ73Z0_152601), the Hungarian Scientific Research Fund grant K-101482, the Deutsche Forschungsgemeinschaft in Sonderforschungsbereich 676, the Post Doctoral Fellowship programme of the Hungarian Academy of Sciences and the Research Funding Program ARISTEIA, HOCTools (co-financed by the European Union (European Social Fund ESF) and Greek national funds through the Operational Program "Education and Lifelong Learning" of the National Strategic Reference Framework (NSRF)).
} 
The t-quark, with the largest mass of known elementary particles, plays an outstanding role in particle physics. From direct measurements the mass of the t-quark is $m_{\mathrm{t}}=173.21 \pm 0.51 \pm$ $0.71 \mathrm{GeV}$ [1], indicating a Yukawa coupling of the t-quark $y_{\mathrm{t}}=0.996 \pm 0.005$. Measuring this mass is important because its value has direct implications on the Higgs sector of the standard model as well as on its extrapolations to high energies, with implications on the stability of the standard model vacuum [2].

The t-quarks decay quickly, almost exclusively into a $W^{+}$boson and a b-quark. Therefore, the t-quarks have to be recontsructed from its decay products, which makes the measurement of $m_{\mathrm{t}}$ highly non-trivial. Several methods have been proposed to measure the mass of the t-quark, summarized in Ref. [3]. One of those is the $m_{\ell b}$ method that extracts $m_{\mathrm{t}}$ from the invariant mass of its decay products, a charged lepton and a $b$-jet [4]. In the dileptonic decay channel of the two $W$ bosons one can use the following estimate for $m_{\mathrm{t}}$ :

$$
m_{\mathrm{est}}^{2}=m_{W}^{2}+\frac{2\left\langle m_{\ell b}^{2}\right\rangle}{1-\left\langle\cos \theta_{\ell b}\right\rangle}
$$

where $\theta_{\ell b}$ is the angle between the charged lepton and the $b$-jet originating from the same t-quark in the rest frame of the $W$ boson. At leading order in perturbation theory $m_{\mathrm{est}}=m_{\mathrm{t}}$.

There can be several possible sources for violation of Eq. (1): (i) higher order corrections both in $t \bar{t}$ production and additional radiation off the top quark decay products, (ii) finite width effects in $t \overline{\mathrm{t}}$ production and finite $\mathrm{b}$-quark mass effects in the decay, (iii) imperfect pairing of the charged lepton and $b$-jet, (iv) acceptance cuts on leptons, jets and missing energy to account for detector effects, (v) experimental issues such as particle misidentification. The first four of these can be studied exactly in perturbative QCD and in fact there are several studies, performed at fixed order, available in the literature that address such questions [5, 6, 7, 8, 9, 10, 11, 12]. A comprehensive summary of the various effects computed at NLO accuracy can be found in Ref. [3]. The bottomline of these theoretically highly challenging computations is that apart from a few observables and in a rather constrained region of the phase space, these effects have been found small, leaving the $t \bar{t}$ production and t-quark decay computed at NLO accuracy and combined in the NWA approximation $[5,6]$ a robust fixed-order prediction.

Another source of uncertainties in the theoretical predictions is the effect of the parton shower and hadronization. In view of the bottom-line found about the uncertainties above, it is important to quantify also these. The up-to-date theoretical tool for making predictions at the hadronic stage of the event evolution is the matching of NLO predictions to shower Monte Carlo programs. At present there exist two methods for achieving such a matching, the MCatNLO [13] and the POWHEG methods $[14,15]$. In this talk we estimate the effects of the parton shower and hadronization by making three different predictions for the $p p \rightarrow \mathrm{t} \overline{\mathrm{t}} \rightarrow W^{+} W^{-} \mathrm{b} \overline{\mathrm{b}}$ process that all include the NLO QCD corrections matched to parton shower using the PowHel framework [16] for generating pre-showered events stored according to the Les-Houches accord [17]. This framework uses the POWHEG method as implemented in POWHEG-BOX [18] and the HELAC-NLO code [19] to provide all matrix elements needed as input by POWHEG-BOX. The details of our computations are presented in [20].

We used for matching the following three types of approximations: (i) The decay chain approximation (DCA), where only the resonant graphs are used, but those are replaced by on-shell 
production of the $t \bar{t}$ pair which is matched to the parton shower such that the decays of the heavy particles are performed by the shower, neglecting both off-shell effects and spin correlations between production and decay. (ii) The narrow-width approximation (NWA) where the on-shell production of the t-quarks is provided at NLO accuracy. Then we push the t-quarks generated in the pre-showered events off-shell according to a Breit-Wigner distribution, followed by a decay at a mixed LO/NLO accuracy [21]. This way we keep only resonant contributions, but effects of finite widths of both t-quarks and $W$ bosons, as well as spin-correlations are included, as implemented in our code Decayer. (iii) The full $W^{+} W^{-} \mathrm{b} \bar{b}$ final state computed at NLO accuracy including both resonant and non-resonant contributions and finite width effects.

With PowHel we can make predictions (a) using the pre-showered events, (b) after parton shower (PS) with stable heavy particles and (c) after full shower Monte Carlo (SMC). Comparing these predictions one can deduce the effect of the PS and that of hadronization and particle decays. In the original publication [20] we made extensive studies of combinations cases (i-iii) with evolution stages $(\mathrm{a}-\mathrm{c})$ and two SMCs (one with two setups) using a set of six standard kinematic distributions, representing three categories: (1) distributions of the b-quarks only, (2) those of the lepton sector, and (3) those involving a b-quark and a charged lepton. In this talk we concentrate on examining the effect of the various approximations (i-iii) and that of hadronization on two distributions: one is the invariant mass of the hardest $\mathrm{b}$-jet and the hardest isolated positron, $m_{\mathrm{b}_{1} e^{+}}$ while the other one is the distribution of the azimuthal separation of the hardest isolated positron and muon $\Delta \phi_{e^{+}} \mu^{-}$. The physical and technical parameters of event generation together with the selection cuts are documented precisely in Ref. [20].

First we compare distributions for the three cases obtained from the pre-showered events. As the NWA approximation with NLO decays is known to describe most kinematic distributions fairly precisely (the inclusive cross section in NWA is about $0.5 \%$ smaller than the prediction of the full calculation) [9], this comparison gives information mainly about the importance of the NLO decays as compared to the LO decays in the two approximations: DCA and the one obtained with the program Decayer, that includes the treatment of both spin correlations and off-shell effects. In order to compare the importance of the SMC effects to the effect of including the decays, we also show the full SMC predictions for case (i) computed with the un-tuned version of the PYTHIA 6.4.28 Monte Carlo [23] (denoted by PY1).

The decays of the heavy particles are well described by both DCA and Decayer up to $150 \mathrm{GeV}$ in the $m_{\mathrm{b}_{1} e^{+}}$-distribution, as seen in Fig. 1.a. For larger values DCA and Decayer fail, leading to an underestimate up to $50 \%$, which is a result of the missing singly- and non-resonant contributions mostly (about $40 \%$ [9]), and of the missing NLO corrections in the decays to much less extent (about an additional $10 \%$ ). Also the two approximate predictions differ in the range of $150-200 \mathrm{GeV}$ due to the off-shell effects in Decayer, missing from the DCA. The effect of the full SMC is small (within $10 \%$ ) below $100 \mathrm{GeV}$ and above $175 \mathrm{GeV}$, but can become very large in between, especially at $150 \mathrm{GeV}$, where the LHE cross section has a sharp drop. As one expects, this sharp drop is smeared by the PS and hadronization (see Fig. 3.a below).

The $\Delta \phi_{e^{+} \mu^{-}}$-distribution, shown in Fig. 1.b, is an example where the differences between the three cases are clearly visible. The prediction by Decayer is within $10 \%$ of the full one, with slightly increasing difference towards the separation of $180^{\circ}$. Nevertheless, the shapes of the LHE predictions are similar, implying that Decayer gives a fairly good approximation to describe spin 
correlations. The difference in normalization is due to the missing NLO corrections in Decayer. The DCA approximation has a different shape due to the absence of spin correlations. For this distribution the full SMC decreases the cross section by about $30 \%$ almost uniformly.
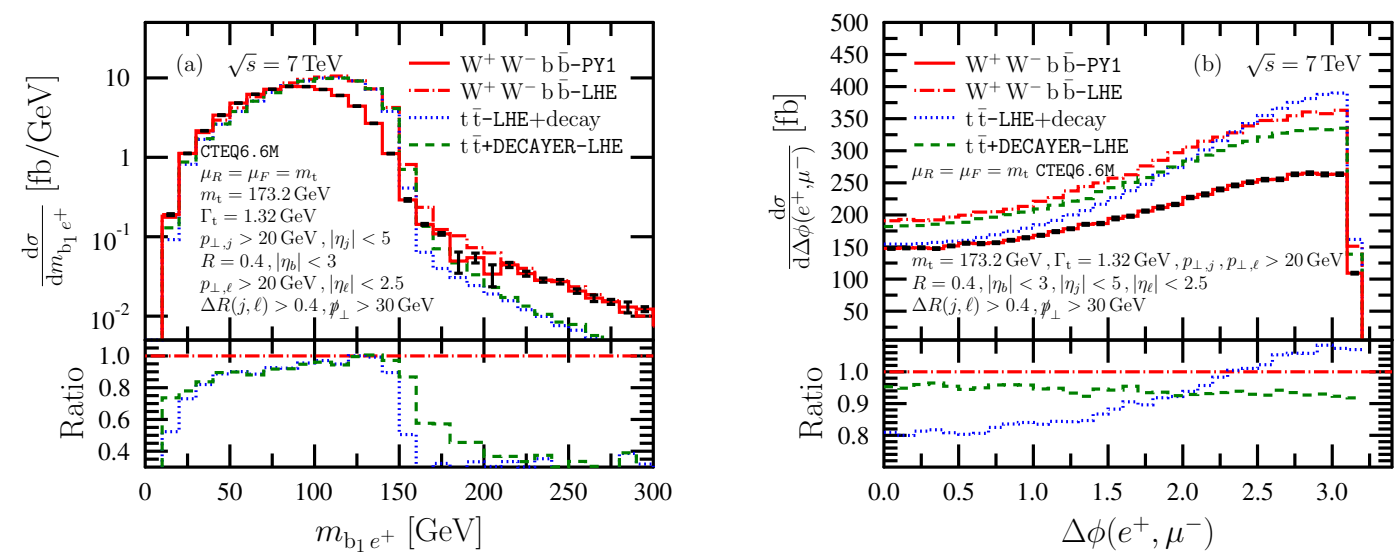

Figure 1: Distributions of a) invariant mass of hardest b-jet and the hardest isolated positron and b) azimuthal separation between the hardest isolated positron and muon for the three cases. The lower inset shows the ratio of the predictions with decay to the $W^{+} W^{-} \mathrm{b} \overline{\mathrm{b}}$-prediction.

Based on the comparison of the three cases of approximations, we conclude the comparison of the three cases of approximations that the predictions with decays give the shapes correctly, the NLO corrections in the decays in general cause only a uniform increase up to $10 \%$ except for distributions involving the charged lepton emerging from the decay of the $W$-bosons (usually the hardest isolated charged lepton). The DCA does not describe the shape of the $\Delta \phi_{e^{+} \mu^{-}}$-distribution due to the lack of spin correlations and both the DCA and the NWA approximations fail in the hard tail of the $m_{\mathrm{b}_{1} e^{+}}$-distribution due to the lack of singly- and non-resonant contributions.

Next, we turn to make predictions at the hadronic stage of event evolution. For this kind of predictions the same selection cuts were applied after shower, hadronization, hadron decay and the application of jet algorithms. We compared almost 50 distributions belonging to the three cases (i-iii). The general trend is that Decayer and DCA give very similar predictions both in shape and normalization for $p_{\perp}$ - and $\eta$-distributions, while the full $W^{+} W^{-} \mathrm{b} \overline{\mathrm{b}}$-computation followed by the SMC differs, but within $10 \%$ of these. These differences are due to the lack of NLO corrections in the decays in the NWA and DCA approximations, and are present already in the distributions of the LHEs, as discussed above (see for instance Fig. 1 below $150 \mathrm{GeV}$ ). The SMC does not change the main features already seen in the LHEs. The $m_{\mathrm{b}_{1} e^{+}}$-distribution shows a similar pattern as seen in the LHEs (cf. Fig. 1) but the large effect of the singly- and non-resonant graphs above $150 \mathrm{GeV}$ becomes much reduced after SMC, and also the sharp drop in the cross section at $150 \mathrm{GeV}$ is smeared (already seen in Fig. 3.a).

In Fig. 2.b we present the $\Delta \phi_{e^{+}} \mu^{-}$-distribution. This distribution is an example where the differences between the three cases were clearly visible in the LHEs. These differences are only slightly altered by the PS, or the full SMC. In particular, the effect of including the spin-correlations leads to an increase of the distribution for small azimuthal separation $\Delta \phi_{e^{+}} \mu^{-}$, where the distribution of case (iii) approaches that of case (ii), both including spin correlations. These are both 15-20\% 
larger than the distribution of case (i), where spin correlation is neglected. At large separations however, after SMC the latter becomes even larger than the predictions from case (iii).
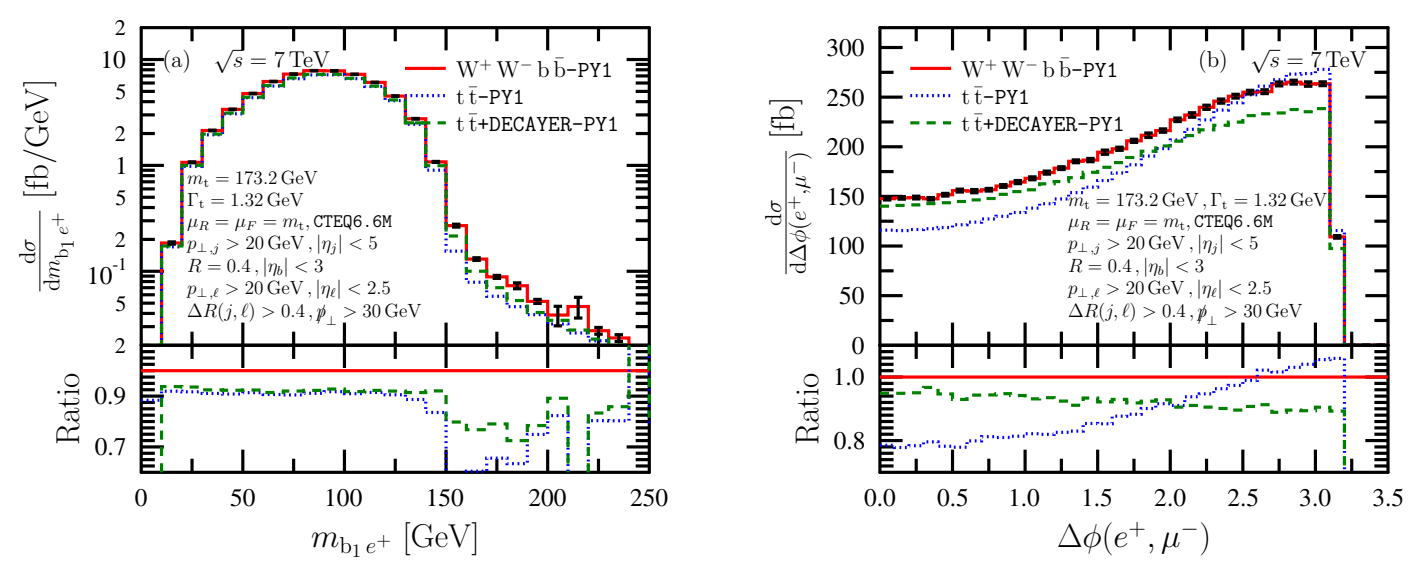

Figure 2: Distributions of a) invariant mass of hardest b-jet and the hardest isolated positron and of b) azimuthal separation between the hardest isolated positron and muon for the three cases. The lower inset shows the ratio of the predictions with $t \bar{t}$-decay (i) and (ii) to that of the complete $W^{+} W^{-} \mathrm{b} \bar{b}$ computation (iii). See text for more details.

Finally, we study the effect of the PS and that of hadronization. In Fig. 3 we show predictions for $W^{+} W^{-} \mathrm{b} \overline{\mathrm{b}}$-production at different stages of event evolution: from pre-showered events (LHEs), after PS and after full SMC. For the PS and SMC we used PY1 (with unstable B hadrons). In addition to the physical selection cuts we employ a jet veto on the non $b$-jets because in the LHEs there can be at most one extra jet besides the $b$ - and $\bar{b}$-jets, while after PS and SMC there are usually many more (less energetic ones). Thus the selection cuts affect the latter much more, decreasing the cross sections significantly (a $10 \%$ decrease of the cross section after PS with the jet veto and very similar decrease is observed on the distributions), which is more a consequence of the selection cuts than the effect of the PS and hadronization. An additional $2 \%$ decrease after full SMC affects the distributions very differently as can be seen in Fig. 3.a where we show the invariant mass of the combination of the hardest $\mathrm{b}$-jet and the hardest isolated positron. For decaying on-shell t-quarks into $W^{+}+\mathrm{b} \rightarrow e^{+} v_{e} \mathrm{~b}$, neglecting the masses of all final decay products, we have

$$
m_{\mathrm{t}}^{2}=p_{\mathrm{t}}^{2}=m_{W^{+}}^{2}+2 p_{e^{+}} p_{\mathrm{b}}+2 p_{v_{e}} p_{\mathrm{b}}
$$

so $m_{e^{+} \mathrm{b}} \leq \sqrt{m_{\mathrm{t}}^{2}-m_{W}^{2}-m_{v_{e} \mathrm{~b}}^{2}}$. Thus, at lowest order in $\mathrm{t} \overline{\mathrm{t}}$-production, there is a strict kinematic limit for the invariant mass of the b-quark and the positron at $\sqrt{m_{\mathrm{t}}^{2}-m_{W}^{2}} \simeq 153 \mathrm{GeV}$, hence the sensitivity to $m_{\mathrm{t}}$. For off-shell t-quarks (e.g. in a computation at NLO accuracy) this kinematic limit is smeared, nevertheless there is a sharp fall of the cross section in the fixed order predictions. As we have shown above the singly- and non-resonant contributions have a significant effect above this limit.

In Fig. 3.a we show that the main effect of the PS and also that of the hadronization is to smear this sharp edge observed in the fixed-order computation, as expected. Apart from this region around $150 \mathrm{GeV}$, the corrections of the SMC are modest. We show the azimuthal separation between the 
hardest isolated positron and muon in Fig. 3.b. For this observable, the main effect of the PS is an almost uniform decrease of about $10 \%$, which is only slightly distorted by the hadronization effects.
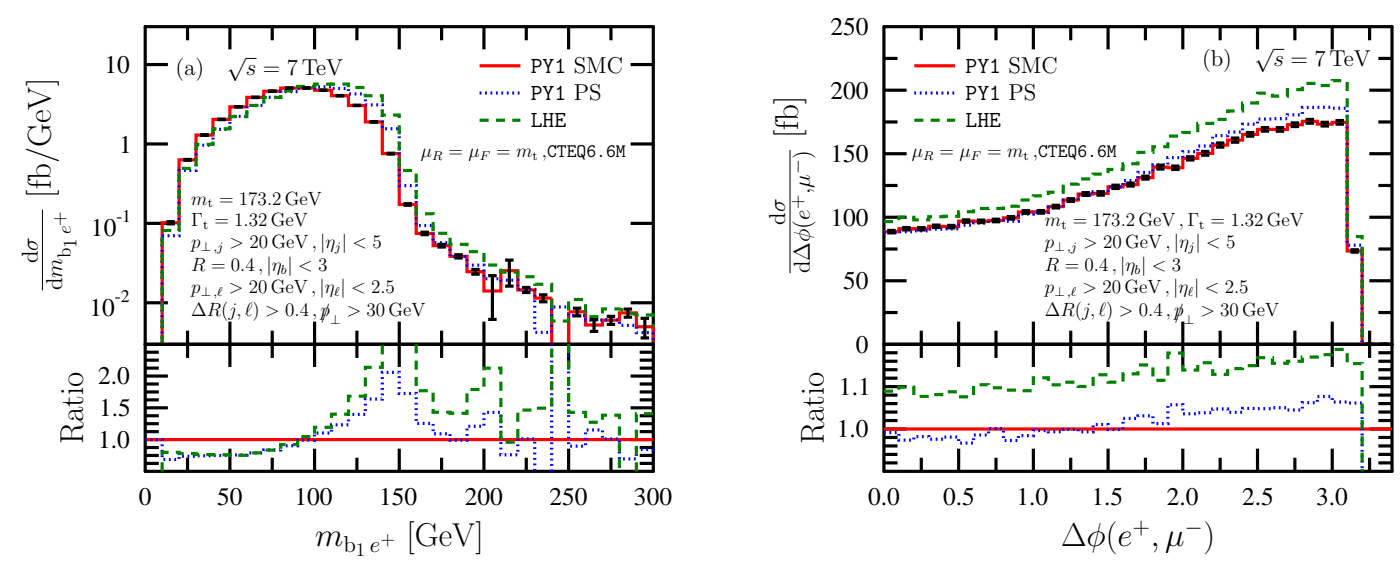

Figure 3: Distributions of a) invariant mass of hardest b-jet and the hardest isolated positron and b) azimuthal separation between the hardest isolated positron and muon after LHE, after PS and after full SMC with PY1. The lower inset shows the ratio of the predictions to the full SMC case.

In this talk we presented predictions for the processes $p p \rightarrow W^{+} W^{-} \mathrm{b} \overline{\mathrm{b}}$ and $p p \rightarrow \mathrm{t} \overline{\mathrm{t}} \rightarrow W^{+} W^{-} \mathrm{b} \overline{\mathrm{b}}$ in the decay-chain and narrow-width approximations at the LHC. We studied and quantified the effects of the approximations on distributions that are the most sensitive (among those we studied) to the non-resonant and spin-correlation effects. We also studied the effect of the parton shower and in general found it small, except in very specific regions of the phase space that do not influence the t-quark mass measurements significantly. We left comparison of our predictions with Decayer to those obtained with a refined version of the POWHEG method with focus on more accurate treatment of radiation off resonances [24, 25] for future work.

This is part of a bigger project with the aim of providing event files produced by PowHel for processes $p p \rightarrow \mathrm{t} \bar{t}+X$, where $X$ is a hard object (jet [26], SM boson [27, 28, 29, 30, 31], b-quark pair [32]). These files are available at http://grid.kfki.hu/twiki/bin/view/DbTheory/ together with the corresponding version of the program.

\section{References}

[1] K. A. Olive et al. [Particle Data Group Collaboration], Chin. Phys. C 38, 090001 (2014).

[2] F. Bezrukov, M. Y. Kalmykov, B. A. Kniehl and M. Shaposhnikov, JHEP 1210, 140 (2012) [arXiv:1205.2893 [hep-ph]].

[3] S. Moch et al., High precision fundamental constants at the TeV scale, arXiv:1405.4781 [hep-ph].

[4] M. Beneke et al., In *Geneva 1999, Standard model physics (and more) at the LHC* 419-529 [hep-ph/0003033].

[5] S. Biswas, K. Melnikov and M. Schulze, JHEP 1008, 048 (2010) [arXiv:1006.0910 [hep-ph]].

[6] J. M. Campbell and R. K. Ellis, J. Phys. G 42, no. 1, 015005 (2015) [arXiv:1204.1513 [hep-ph]].

[7] A. Denner, S. Dittmaier, S. Kallweit, and S. Pozzorini, Phys.Rev.Lett. 106 (2011) 052001, [arXiv:1012.3975 [hep-ph]]. 
[8] G. Bevilacqua, M. Czakon, A. van Hameren, C. G. Papadopoulos, and M. Worek, JHEP 02 (2011) 083, [arXiv:1012.4230 [hep-ph]].

[9] A. Denner, S. Dittmaier, S. Kallweit and S. Pozzorini, JHEP 1210, 110 (2012) [arXiv:1207.5018 [hep-ph]].

[10] F. Cascioli, S. Kallweit, P. Maierhöfer and S. Pozzorini, Eur. Phys. J. C 74, no. 3, 2783 (2014) [arXiv:1312.0546 [hep-ph]].

[11] R. Frederix, Phys. Rev. Lett. 112, no. 8, 082002 (2014) [arXiv:1311.4893 [hep-ph]].

[12] G. Heinrich, A. Maier, R. Nisius, J. Schlenk, and J. Winter, JHEP 1406, 158 (2014) [arXiv:1312.6659 [hep-ph]].

[13] S. Frixione and B. R. Webber, JHEP 0206, 029 (2002) [hep-ph/0204244].

[14] P. Nason, JHEP 0411, 040 (2004) [arXiv:hep-ph/0409146].

[15] S. Frixione, P. Nason and C. Oleari, JHEP 0711, 070 (2007) [arXiv:0709.2092].

[16] M. V. Garzelli, A. Kardos and Z. Trócsányi, PoS EPS -HEP2011, 282 (2011) [arXiv:1111.1446 [hep-ph]].

[17] J. Alwall et al., Comput. Phys. Commun. 176, 300 (2007) [arXiv:hep-ph/0609017].

[18] S. Alioli, P. Nason, C. Oleari and E. Re, JHEP 1006, 043 (2010) [arXiv:1002.2581].

[19] G. Bevilacqua, M. Czakon, M. V. Garzelli, A. van Hameren, A. Kardos, C. G. Papadopoulos, R. Pittau and M. Worek, Comput. Phys. Commun. 184, 986 (2013) [arXiv:1110.1499 [hep-ph]].

[20] M. V. Garzelli, A. Kardos and Z. Trócsányi, JHEP 1408, 069 (2014) [arXiv:1405.5859 [hep-ph]].

[21] S. Frixione, E. Laenen, P. Motylinski and B. R. Webber, JHEP 0704, 081 (2007) [hep-ph/0702198 [HEP-PH]].

[22] M. Cacciari, G. P. Salam and G. Soyez, Eur. Phys. J. C 72, 1896 (2012) [arXiv:1111.6097 [hep-ph]], http://fastjet.fr

[23] T. Sjostrand, S. Mrenna and P. Z. Skands, JHEP 0605, 026 (2006) [arXiv:hep-ph/0603175].

[24] J. M. Campbell, R. K. Ellis, P. Nason and E. Re, JHEP 1504, 114 (2015) [arXiv:1412.1828 [hep-ph]].

[25] T. Jezo and P. Nason, arXiv:1509.09071 [hep-ph].

[26] A. Kardos, C. Papadopoulos and Z. Trócsányi, Phys. Lett. B 705, 76 (2011) [arXiv:1101.2672 [hep-ph]].

[27] M. V. Garzelli, A. Kardos, C. G. Papadopoulos and Z. Trócsányi, Europhys. Lett. 96, 11001 (2011) [arXiv:1108.0387 [hep-ph]].

[28] M. V. Garzelli, A. Kardos, C. G. Papadopoulos and Z. Trócsányi, Phys. Rev. D 85, 074022 (2012) [arXiv:1111.1444 [hep-ph]].

[29] M. V. Garzelli, A. Kardos, C. G. Papadopoulos and Z. Trócsányi, JHEP 1211, 056 (2012) [arXiv:1208.2665 [hep-ph]].

[30] A. Kardos and Z. Trócsányi, JHEP 1505, 090 (2015) [arXiv:1406.2324 [hep-ph]].

[31] A. Kardos and Z. Trócsányi, Nucl. Phys. B 897, 717 (2015) [arXiv:1408.0278 [hep-ph]].

[32] M. V. Garzelli, A. Kardos and Z. Trócsányi, JHEP 1503, 083 (2015) [arXiv:1408.0266 [hep-ph]]. 